\title{
Composición y diversidad florística de los páramos en la Reserva de Producción de Fauna Chimborazo, Ecuador
}

\author{
(Composition and diversity of High Andean in the Fauna \\ Production Reserve Chimborazo, Ecuador)
}

\author{
Jorge Caranqui ${ }^{1}$, Patricio Lozano ${ }^{1}$, Julio Reyes ${ }^{1}$
}

\begin{abstract}
Resumen:
El presente estudio indaga la diversidad florística de 9 zonas de muestreo en cuatro parcelas de 1 $\mathrm{m}^{2}$ de páramos en varias localidades de la Reserva de Producción de Fauna Chimborazo. Para el desarrollo del presente estudio utilizamos una adaptación del método de parcelas GLORíA. Se obtuvieron las coberturas(\%) en cada una de las parcelas, además de los índices de diversidad y similitud con el respectivo análisis. Los datos obtenidos reflejan una diversidad que puede ir de media a baja, se cree que esto se debe a las actividades antropogénicas que se han realizado en estas formaciones vegetales. Con la presencia mayoritariamente de Calamagrostis intermedia, se podría establecer que los tipos de vegetación existentes en mayor porcentaje es páramo herbáceo; es la especie que casi siempre está presente en la mayoría de tipos de vegetación de la RPF Chimborazo y con una dominancia alta que influye en los resultados de índices de diversidad florística bajos que se encontró en el análisis. Como consecuencia la Familia más abundante es Poaceae además de Asteraceae.
\end{abstract}

Palabra clave: Páramo herbáceo, Diversidad, Similitud, Calamagrostis, Poaceae, Asteraceae

\begin{abstract}
:
The present study inquire the floristic diversity of 9 sampling in four plots of $1 \mathrm{~m}^{2}$ of high andean in several locations in the "Reserva de Producción de Fauna Chimborazo". For the development of this study, we used an adaptation of the method of plots "Gloria". With coverage (\%) in each of the plots, Further the diversity indices and similarity with respective analysis were obtained. The data obtained reflect a diversity that can range from medium to low, believe that this is due to anthropogenic activities that have taken place in these ecosistems. With the presence mostly Calamagrostis intermedia, it could establish that the type of vegetation is herbaceous in high andean is higher percentage; is the species that is almost always present in most types of vegetation of the RPF Chimborazo and high dominance that influences the results of low floristic diversity indices was found in the analysis. As a result the most abundant family Asteraceae is well Poaceae.
\end{abstract}

Keywords: herbaceous páramos, Diversity, Similarity, Calamagrostis, Poaceae, Asteraceae

\footnotetext{
${ }^{1}$ Escuela Superior Politécnica del Chimborazo, Riobamba - Ecuador (jorge.caranqui@espoch.edu.ec)
} 


\section{Introducción}

Los ecosistemas de páramo son categorizados como uno de los sitios con mayor biodiversidad del mundo, considerado por algunos como archipiélago biológico (Pauli et-al, 2003), (Azócara, 1981). En el Ecuador la franja altitudinal entre los 3000 hasta los 4500 m s.n.m. contiene casi el 30\% de las especies de plantas vasculares (Josse et-al, 2000), esto demuestra la gran representatividad que tiene este ecosistema y la importancia de conocer cuál es su diversidad florística, ya que esta clase de información servirá como punto de partida para el monitoreo del cambio climático y el comportamiento de la flora.

Según Mena y Hofstede ( 2006), el páramo en realidad posee una variedad mucho mayor de lo que la imagen clásica ("lugar yermo desprovisto de árboles") nos haría pensar. Los páramos, en el Neotrópico, cubren alrededor del $2 \%$ de la superficie de los paises de esa región; tiene cerca de 125 familias, 500 géneros y 3400 especies de plantas vasculares. En términos del Ecuador, aún no se conoce el número exacto de especies de plantas que viven en los páramos, pero (LeónYánez, 2000), sugiere que son alrededor de 1.500 especies. También Sklenar et-al. (2005), manifiesta que hasta la fecha, se han registrado para los páramos del Ecuador un total de 1.524 especies, siendo para este ecosistema y en relación a su tamaño, el país con la flora más diversa de la región andina. De las especies registradas para Ecuador, se estima que alrededor de 628 especies de plantas son endémicas, esto equivale al 15\% de toda la flora endémica del país y el $4 \%$ del total de su flora (Hofstede et-al, 2014). De acuerdo a León-Yánez (2011) el 75\% de éstas especies endémicas están amenazadas y apenas el $48 \%$ están dentro de áreas protegidas.

En el Ecuador los páramos ocupan una extensión de 1'337.119, que corresponden aproximadamente al $5 \%$ de la extensión territorial. La provincia de Chimborazo, con una extensión de 648.124 has, posee más de 236.000 has de ecosistema de páramo, es decir, el 36,9\% de la superficie de la provincia y otras 83.800 has son bosque andino y alto andino, es decir, el 13,1\% de la superficie de la provincia (Bustamante, Albán, y Arguello, 2011). A nivel nacional, esto representa el 17,7\% del total de superficie de páramo en el país (Beltrán et-al, 2009; Beltrán, 2010). Adicionalmente, en la provincia existen dos áreas protegidas del Patrimonio Nacional del Estado (PANE), la Reserva de Producción de Fauna Chimborazo y el Parque Nacional Sangay, entre ellas protegen 91.667 has que representan el 14\% del total de la provincia y el $31 \%$ del total de los páramos de la provincia. Aunque estas áreas se encuentran bajo un régimen de protección, varias áreas dentro de ellas muestran un alto grado de intervención (Rojas, 2010; Salgado y Cárate 2010).

Las áreas protegidas constituyen el principal instrumento de conservación que salvaguarda en la actualidad el $14 \%$ de la superficie terrestre (incluida la Antártida) y el 3,41\% de la superficie 
marina del planeta, a través de 209.428 áreas, en 32 '868.673 km2 (Deguignet, y otros, 2014). El objetivo primario que orienta la gestión in situ de éstos espacios es la conservación a largo plazo de la naturaleza, de sus servicios ecosistémicos y sus valores culturales asociados (UICN, 2008). La Reserva de Producción FaunistícaChimborazo es parte de las 51 áreas que conforman el Sistema Nacional de Áreas Protegidas del Ecuador, que representa aproximadamente el $20 \%$ del territorio nacional, fue creada con acuerdo Ministerial No. 437 el 26 de octubre de 1987, localizado en las provincias de Chimborazo, Bolívar y Tungurahua (Ministerio de Ambiente, 1995). Su extensión es de 52.683 has; su altitud varía desde los 3650 a 6310 m.s.n.m.; presenta un clima frio andino con temperaturas desde $-4.8^{\circ} \mathrm{C}$ hasta los $11.4^{\circ} \mathrm{C}$; registra una precipitación de 809 $\mathrm{mm}$ en las zonas menos lluviosas y $1300 \mathrm{~mm}$ en las zonas más húmedas, y sus objetos de conservación son los páramos, sistemas hídricos y humedales, bosques y arbustales siempre verdes, y sitos arqueológicos (EcoCiencia, 2014).

Entendiendo este contexto, el objetivo del presente estudio es cuantificar y analizar la diversidad y similitud florística de los páramos en la Reserva de Producción de Fauna Chimborazo.

\section{Métodos}

\section{1. Área de estudio}

El estudio se realizó en la Reserva de Producción de Fauna Chimborazo, perteneciente a páramos herbáceos (Sierra, 1999) en la cual se identificó 9 zonas de muestreo (Tabla 1 y Figura 1). Estas zonas de muestreo están localizadas en 3 provincias, 4 cantones, y 5 parroquias de la referida área protegida (Figura 1).

Tabla 1. Ubicación de las localidades dentro de la R.F.Chimborazo

\begin{tabular}{|l|l|l|l|l|l|l|}
\hline LOCALIDAD & PARROQUIA & CANTÓN & PROVINCIA & $\begin{array}{l}\text { ALTITUD } \\
\text { (msnm.) }\end{array}$ & LATITUD & LONGITUD \\
\hline Ganquis & San Juan & Riobamba & Chimborazo & 3690 & $1^{\circ} 31^{\prime} 39^{\prime \prime} \mathrm{S}$ & $78^{\circ} 52^{\prime} 02^{\prime \prime} \mathrm{W}$ \\
\hline $\begin{array}{l}\text { Condor } \\
\text { Chamana }\end{array}$ & San Andrés & Guano & Chimborazo & 3745 & $1^{\circ} 28^{\prime} 34^{\prime \prime} \mathrm{S}$ & $78^{\circ} 44^{\prime} 50^{\prime \prime} \mathrm{W}$ \\
\hline Ruta del Hielero & San Juan & Riobamba & Chimborazo & 4070 & $1^{\circ} 30^{\prime} 18^{\prime \prime} \mathrm{S}$ & $78^{\circ} 47^{\prime} 27^{\prime \prime} \mathrm{W}$ \\
\hline Mechahuasca 1 & Pilahuín & Ambato & Tungurahua & 4190 & $01^{\circ} 24^{\prime} 10^{\prime S}$ & $78^{\circ} 48^{\prime} 13^{\prime \prime} \mathrm{W}$ \\
\hline Río Blanco & Pilahuín & Ambato & Tungurahua & 4242 & $01^{\circ} 22^{\prime} 34 \mathrm{~S}$ & $78^{\circ} 47^{\prime} 24^{\prime \prime} \mathrm{W}$ \\
\hline Mechahuasca 2 & Pilahuín & Ambato & Tungurahua & 4190 & $01^{\circ} 25^{\prime} 29^{\prime \prime} \mathrm{S}$ & $78^{\circ} 47^{\prime} 53^{\prime \prime} \mathrm{W}$ \\
\hline El Sinche & Facundo Vela & Guaranda & Bolívar & 3990 & $01^{\circ} 26^{\prime} 07^{\prime \prime} \mathrm{S}$ & $78^{\circ} 56^{\prime} 43 \mathrm{~W}$ \\
\hline Arenal & San Juan & Riobamba & Chimborazo & 4100 & $01^{\circ} 21^{\prime} 59^{\prime \prime S}$ & $78^{\circ} 52^{\prime} 48^{\prime \prime} \mathrm{W}$ \\
\hline Mechahuasca 3 & Pilahuín & Ambato & Tungurahua & 4190 & $01^{\circ} 25^{\prime} 07^{\prime \prime} \mathrm{S}$ & $78^{\circ} 47^{\prime} 30^{\prime \prime} \mathrm{W}$ \\
\hline
\end{tabular}




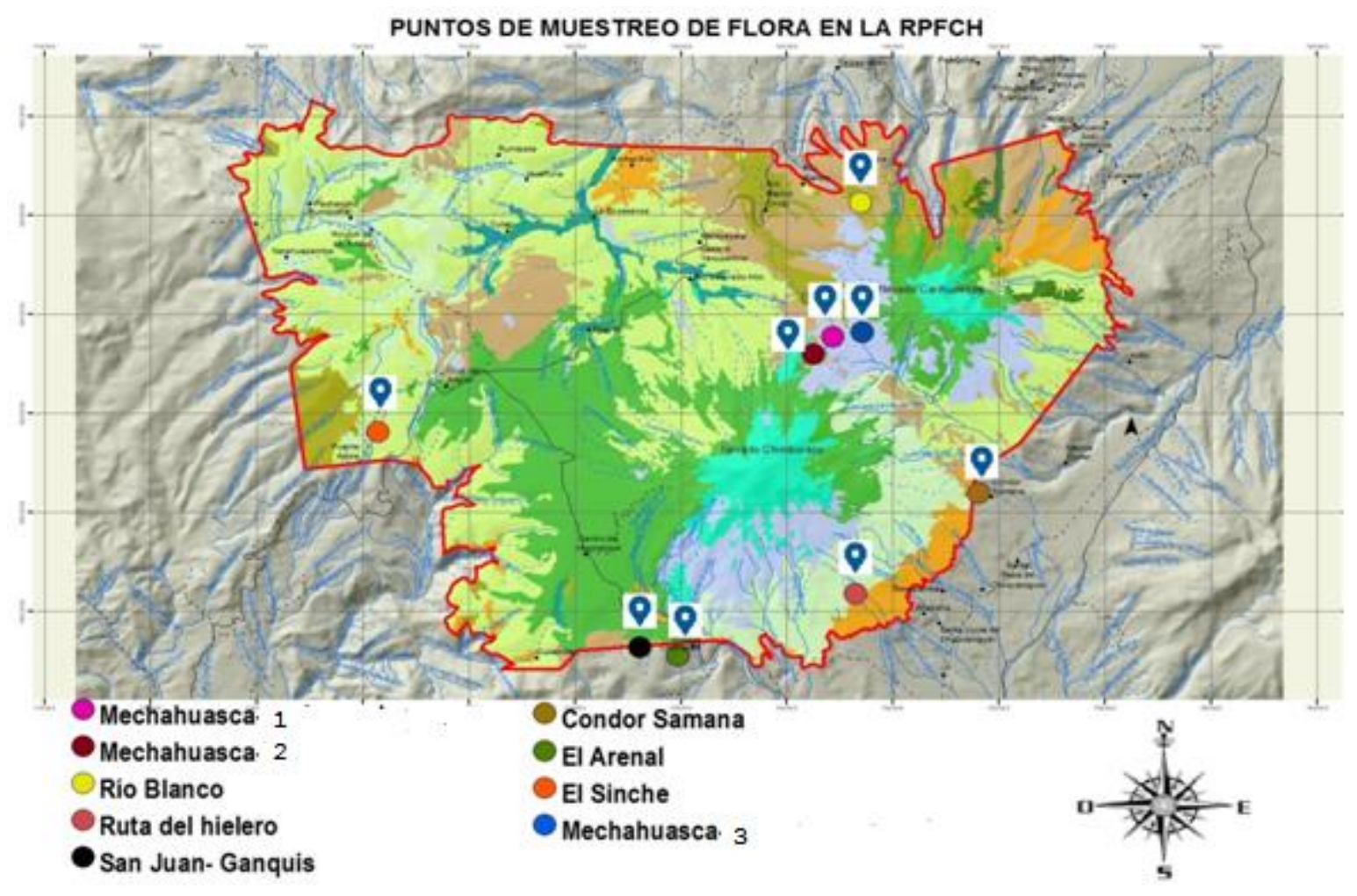

Figura 1. Ubicación de las localidades dentro de la R.F.Chimborazo

\subsection{Diseño de muestreo}

La metodología utilizada fue la propuesta por (Pauli et-al, 2003), para el proyecto GLORIA en la región europea, razón por la cual se hicieron algunas modificaciones que permitieron adaptarla a los páramos andinos (Suarez et-al, 2012) (Eguiguren et-al, 2010). Las zonas de muestreo se identificaron aplicando criterios de tipo de vegetación, composición vegetal y estado de conservación. En cada zona identificada se instaló un cuadrante de $5 \times 5 \mathrm{~m}$, que se utilizó para el muestreo de la vegetación. Cada cuadrante se subdividió en parcelas de $1 \times 1 \mathrm{~m}$., las observaciones de vegetación se llevó a cabo únicamente en las cuatro parcelas de las esquinas o extremos, ya que los otros pueden quedar alterados por el pisoteo de los investigadores a lo largo del muestreo. Cada uno de las parcelas de $1 \times 1 \mathrm{~m}$. se subdividieron en cuadriculas de $0.1 \times 0.1 \mathrm{~m}$, para ello se utilizó un armazón de tubería PVC con un enrejado formado por hilos finos que delimitan 100 celdillas de 0,1 x 0,1 m., de acuerdo a la metodología del manual GLORIA adaptado por (Rodríguez, 2011) y (Caranqui et-al, 2013), esto permitió obtener la mayor cantidad de información para su posterior análisis.

Dentro de las parcelas se registró información referente al número de especies y la cobertura de cada una de ellas, esto sirvió para determinar la diversidad por familia, densidad, e índice de diversidad. Para mayor información de las especies monitoreadas se revisó en (Jorgensen \& Yánez, 1999) y la actualización se consultó en Missouri Botanical Garden (2015) 


\subsection{Análisis de datos}

Se generó un listado de especies con sus respectivas coberturas en cada una de las parcelas, con los cuales se obtuvo: riqueza, diversidad (índice de Simpson), similitud (índice de Bray Curtis), calculados en el software estadístico PAST.

Según (Golicher, 2012), la fórmula para el índice de Simpson es:

$$
D=\frac{\sum_{i=1}^{S} n_{i}\left(n_{i}-1\right)}{N(N-1)}
$$

Donde $S$ es el número de especies, $N$ es el total de organismos presentes (o unidades cuadradas) y $n$ es el número de ejemplares por especie.

En la ecología y la biología, la disimilitud Bray-Curtis (1957), es una estadística que se usa para cuantificar la disimilitud en la composición entre dos sitios diferentes, basadas en conteos en cada sitio. Según la definición de Bray y Curtis, el índice de similitud:

$$
B C_{i j}=\frac{2 C_{i j}}{S_{i}+S_{j}}
$$

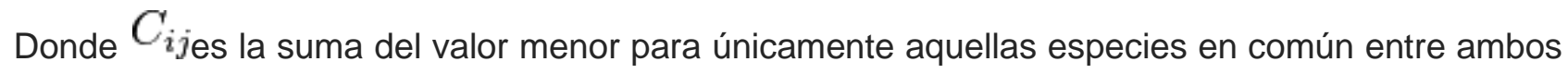

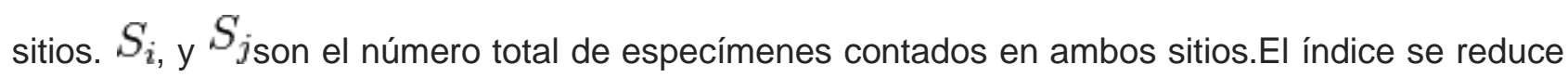
a $2 \mathrm{C} / 2$ = C, donde la abundancia en cada sitio se expresan como un porcentaje. El tratamiento adicional se puede encontrar en Legendre y Legendre. La disimilitud de Bray-Curtis está directamente relacionada con el índice de similitud de Sørensen $Q S_{i j}$ entre los mismos sitios:

$$
\overline{B C}_{i j}=1-Q S_{i j} .
$$

La disimilitud de Bray-Curtis está obligado entre 0 y 1 , donde 1 significa que los dos sitios tienen la misma composición (es decir que comparten todas las especies), y 0 significa que los dos sitios no comparten ninguna especie. En los sitios con donde $A C$ es intermedia (por ejemplo, $A C=0,5$ ) este índice se diferencia de otros índices comúnmente utilizados.

\section{Resultados}

\subsection{Riqueza Florística y Cobertura}

Se encontró en total 20 familias, 36 géneros y 46 especies distribuidas en las 9 zonas de muestreo de la RPF Chimborazo (Figura 1). Las familias con mayor número de especies son Asteraceae (9), Poaceae y Geraniaceae con 5; la mayoría de géneros corresponden a una sola especie a excepción de Geranium y Lachemilla.

Calamagrostis intermedia tiene presencia en las 8 de las 9 zonas de muestreo y sus coberturas en 
3 zonas superan la mitad de la cobertura total, Phyllactis rigida y Eryngium humile tienen presencia en 5 de las 9 zonas, sin embargo Phyllactis rigida tiene mayor cobertura.

Hypochaeris sessiliflora se encuentra en 4 de las 9 zonas de muestreo pero en menor cobertura. El resto de especies tienen presencias y coberturas quen son de menor representatividad (Tabla 2).

Tabla 2. Listado de especies más representativas con sus respectivas coberturas (400\%) en las 22 parcelas.

\begin{tabular}{|c|c|c|c|c|c|c|c|c|c|}
\hline ESPECIES & GA & $\mathrm{CCH}$ & $\mathbf{R H}$ & RB & MCH1 & SI & ARE & $\mathrm{MCH} 2$ & MCH3 \\
\hline $\begin{array}{l}\text { Calamagrostis intermedia (J.Presl) } \\
\text { Steud. }\end{array}$ & 365 & 285 & 75 & 71 & 164 & 250 & 14 & 26 & 0 \\
\hline Phyllactis rigida (Ruíz \& Pav.) Pers. & 0 & 0 & 15 & 49 & 0 & 115 & 0 & 240 & 185 \\
\hline Eryngium humile Cav. & 10 & 0 & 0 & 67 & 128 & 3 & 0 & 0 & 40 \\
\hline Xenophyllum humile (Kunth) V.A.Funk & 0 & 0 & 168 & 0 & 0 & 0 & 0 & 0 & 0 \\
\hline Geranium sibbaldiodes Benth & 0 & 0 & 0 & 121 & 0 & 0 & 0 & 0 & $\underline{0}$ \\
\hline Astragalus geminiflorus Bonpl. & 0 & 0 & 0 & 0 & 0 & 0 & 65 & 0 & 0 \\
\hline Loricaria illinisae (Benth)) Cuatrec. & 0 & 0 & 4 & 0 & 0 & 0 & 0 & 53 & 0 \\
\hline Baccharis caespitosa (Ruíz \& Pav.) Pers. & 0 & 4 & 0 & 0 & 0 & 0 & 31 & 20 & $\underline{0}$ \\
\hline Agrostis breviculmis Hitchc. & 0 & 0 & 0 & 0 & 0 & 7 & 0 & 0 & 45 \\
\hline Paspalum sp. & 0 & 0 & 0 & 0 & 50 & 0 & 0 & 0 & 0 \\
\hline Hypochaeris sessiliflora Kunth & 0 & 0 & 39 & 2 & 2 & 0 & 2 & 0 & 0 \\
\hline Isolepis inundata R.Br. & 0 & 0 & 0 & 0 & 0 & 0 & 0 & 0 & 45 \\
\hline Plantago linearis Kunth & 0 & 0 & 0 & 0 & 0 & 0 & 0 & 0 & 40 \\
\hline $\begin{array}{l}\text { Breutelia tomentosa (Sw. Ex Brid.) } \\
\text { A.Jaeger }\end{array}$ & 0 & 30 & 3 & 0 & 0 & 0 & 0 & 0 & $\underline{0}$ \\
\hline Werneria pigmae Gillis ex Hook. \& Arn. & 0 & 0 & 0 & 0 & 0 & 0 & 0 & 33 & 0 \\
\hline $\begin{array}{l}\text { Lachemilla aphanoides (Mutis ex L.f.) } \\
\text { Roethm. }\end{array}$ & 2 & 3 & 25 & 0 & 0 & 0 & 0 & 0 & 0 \\
\hline Distichia muschoides Nees \& Meyen & 0 & 0 & 0 & 25 & 0 & 0 & 0 & 0 & 5 \\
\hline Campylopus sp. & 0 & 2 & 0 & 19 & 1 & 0 & 0 & 7 & 0 \\
\hline Geranium diffusum Kunth & 0 & 24 & 0 & 0 & 0 & 0 & 0 & 0 & 0 \\
\hline Werneria nubigena Kunth & 0 & 0 & 0 & 0 & 0,5 & 0 & 4 & 11 & 0 \\
\hline Valeriana microphylla Kunth & 0 & 15 & 0 & 0 & 0 & 0 & 0 & 0 & 0 \\
\hline $\begin{array}{l}\text { Lachemilla orbiculata (Ruíz \& Pav.) } \\
\text { Rydb. }\end{array}$ & 4 & 4 & 0 & 0 & 0 & 6 & 0 & 0 & 0 \\
\hline Gamochaeta americana (Mill.) Wedd. & 0 & 0 & 0 & 0 & 0 & 12 & 0 & 0 & 0 \\
\hline $\begin{array}{l}\text { Huperzia crassa (Humb. \& Bonpl. Ex } \\
\text { Willd.) Rothm. }\end{array}$ & 0 & 0 & 0 & 8 & 0 & 0 & 0 & 0 & $\underline{0}$ \\
\hline Chuquiraga jusseiu J.F.Gmel. & 0 & 0 & 0 & 0 & 0 & 0 & 7 & 0 & 0 \\
\hline
\end{tabular}

GA: Ganquis; CCH: Cóndor Chamana; RH: Ruta del hielero; RB: Río Blanco; MCH1: Mechahuasca pajonal; SI: El Sinche; ARE: Arenal; $\mathbf{M C H 2 : ~ M e c h a h u a s c a ~ m o s a i c o ; ~} \mathbf{M C H}$ 3:Mechahuasca humedal. 


\subsection{Diversidad}

El listado de especies (Tabla 2) se ingresó en el programa PAST generándose el índice de diversidad de Simpson que fluctúa de 0.17- 0.79 (tabla 2), el cual resulta que los valores varían de bajo a medio en base al número de especies y cobertura (\%) en cada parcela ya que todos los valores no se acercan a 1, el valor más próximo a 1 es Río Blanco con 0,79; el resto no supera 0,61. El número de especies varía de 8 a 12 especies y correspondientemente las que tienen la mayor cantidad de especies tienen alto índice de diversidad como son Río Blanco (RB) y Ruta del Hielero $(\mathrm{RH})$. Hay índices bajos de diversidad en algunas zonas de muestreo, especialmente en Ganquis (GA) se obtuvo 0.169 y probablemente se deba a la fuerte dominancia de Calamagrostis intermedia, y que además presenta una tendencia similar en el resto de zona.

Tabla 3. Datos de los nueve zonas de muestreo de taxones, abundancia y diversidad.

\begin{tabular}{|l|r|r|r|r|r|r|r|r|r|}
\hline & GA & CCH & RH & RB & MCH1 & SI & ARE & MCH2 & MCH3 \\
\hline Especies & 10 & 11 & 9 & 11 & 12 & 8 & 7 & 9 & 8 \\
\hline Altitud (m) & 3690 & 4050 & 4070 & 4242 & 4190 & 3990 & 4200 & 4000 & 4000 \\
\hline Cobertura (\%) & 400 & 371,5 & 331,5 & 364 & 354,5 & 400 & 126 & 398 & 365 \\
\hline I.Simpson & 0,1691 & 0,4568 & 0,6145 & 0,7989 & 0,5559 & 0,563 & 0,6044 & 0,5998 & 0,6846 \\
\hline
\end{tabular}

GA: Ganquis; CCH: Cóndor Chamana; RH: Ruta del hielero; RB: Río Blanco; MCH1: Mechahuasca pajonal; SI: El Sinche; ARE: Arenal; MCH2: Mechahuasca mosaico; MCH3:Mechahuasca humedal.

\subsection{Similitud}

Los clusters, presentados en la Figura 2, generaron 3 grupos, el primero corresponde a Mechahusca $2(\mathrm{MCH} 2)$ y Mechahusca $3(\mathrm{MCH} 3)$ que corresponde a una mezcla de especies de almohadillas con especies de húmedales con una dominancia de Phyllactis rigida y ausencia o presencia menor de Calamagrostis intermedia; el segundo grupo está Río Blanco (RB), Mechahusca Pajonal (MCH1), El Sinche (SIN), y Ganquis (GA) donde hay presencia de Calamagrostis intermedia y por tal razón existen subgrupos como el que forman Río Blanco (RB) con un menor porcentaje de Calamagrostis intermedia; Mechahusca Pajonal (MCH1) con un porcentaje intermedio y finalmente El Sinche (SIN) y Ganquis (GA) que tienen los mayores porcenatjes de cobertura de la Poaceae. Y el tercero Arenal (ARE) a super páramo que si bien tiene menor cobertura que el resto su distribución de especies es mas homogenea.

\section{Discusión}

Las Familias más representativas en este estudio fueron Poaceae, Asteraceae y Geraniaceae que tuvieron la mayor cantidad de especies; además Poaceae contiene a Calamagrostis intermedia que fue la especie que tuvo los mayores valores cuantitativos en la mayoría de zonas de muestreo, seguido de Caprifoliaceae con Phyllactis rigida que fue la segunda con mayores valores. 


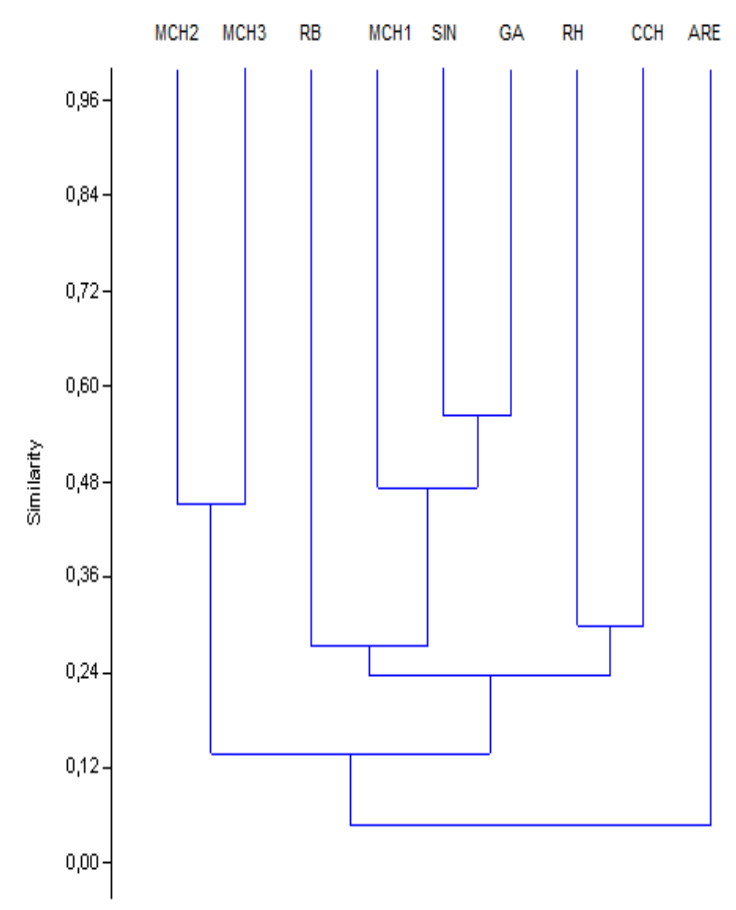

Figura 2. Cluster del índice de Bray Curtis con los 13 parcelas

GA: Ganquis; CCH: Cóndor Chamana; RH: Ruta del hielero; RB: Río Blanco; MCH1: Mechahuasca pajonal; SI: El Sinche; ARE: Arenal; MCH2: Mechahuasca mosaico; MCH3:Mechahuasca humedal.

De los pocos estudios que se han realizado en la RPF Chimborazo existe información cualitativa (Familias y especies) y la cuantitativa (abundancia/ riqueza) varía en la metodología utilizada (Salgado et-al, 2011); (Salgado y Cárate, 2010) y (Beltrán, 2009), por tanto, mal podríamos tratar de comparar. Sin embargo, en cuanto a los resultados cualitativos del Ministerio de Ambiente (2013) y (EcoCiencia, 2012), estos hacen referencia a las familias y taxones citados también en este trabajo y coinciden de forma general.

De las nueve zonas de muestreo, en ocho hay presencia de Calamagrostis intermedia y en la que mayor porcentaje se encuentra es en el sector de Ganquis, este resultado coincide con el estudio de Caranqui (2013), en cuanto a la presencia característica de la especie en la RPF Chimborazo y la representatividad de la Familia Poaceae en este tipo de formaciones vegetales.

El índice de diversidad de Simpson (Tabla 3) indica la relación entre riqueza o número de especies y la abundancia o número de individuos por especies en cualquier sitio dado (Smith \& Smith, 2001). En el presente estudio se encontraron hasta 12 especies por zona de muestreo. Este resultado conjuntamente con los ya mencionados evidencian que la diversidad florística es media a baja, lo cual coinciden con algunos estudios realizados en la provincia como el de Caranqui et-al(2013). 
Podríamos decir que, la presencia de un mayor o menor número de especies está influenciado tal vez por el estado de conservación de los páramos o por el grado de intervención de estos tipos de vegetación. La mayoría de los páramos de pajonal son quemados anualmente, o por lo menos cada ciertos años, por incendios producidos deliberadamente por los pobladores, con el fin de obtener pastizales para el ganado vacuno y ovejero. Por lo tanto, todos los taxones de plantas del páramo, poseen adaptaciones que les permite sobrevivir los frecuentes incendios (Laaegard, 1992). Éstas adaptaciones deben haber evolucionado mucho antes que los incendios antropogénicos tuvieran un impacto en los páramos, más o menos dentro de los últimos 10.000 años. Las características morfológicas y fisiológicas que permiten a las plantas del páramo sobrevivir los frecuentes incendios probablemente evolucionaron como adaptaciones a otros factores como la sequía y las fluctuaciones de temperaturas diurnas.

Además de factores bióticos, se suma el aspecto antrópico, el cual en la provincia de Chimborazo ha tenido un papel muy importante en los cambios y transformaciones sobre la estructura y composición de este ecosistema andino. La mayoría de autores están de acuerdo que el páramo de pajonal está sumamente influenciado por las actividades humanas, en particular por los incendios causados por el hombre (Laaegard, 1992). Entonces podríamos inferir que los valores bajos de diversidad y la mayor presencia de familias como Poaceae y especies como Calamagrostis intermedia se deberían a lo explicado en los párrafos anteriores.

\section{Conclusiones y Recomendaciones}

La mayoría de parcelas según el índice de diversidad de Simpson tienen en su mayoría valores de medio a bajo que fluctúa de: 0.17-0.79. Los valores bajos que se obtuvieron se presume que es a la alta dominancia que se encontró en la mayoría de parcelas por parte de Calamagrostis intermedia.

Con los resultados presentados la familia Poaceae y Asteraceae tienen los mayores valores y número de especies y coinciden con los estudios cualitativos de este tipo de ecosistema. Y además inferimos que son los grupos botánicos que mas resisten a las condiciones climáticas adversas y a los procesos de degradación del páramo en la Reserva.

Se recomienda que se respeten los procesos naturales de regeneración que se producen en el Páramo y que se haga campañas para evitar las actividades antropogénicas especialmente la quema del pajonal que es el problema más grave en la RFPCH. 


\section{Agradecimientos}

A la Dirección provincial del Ambiente, en la persona del Ing. Marcelo Pino, por ayudarnos en la logística en la RPF Chimborazo. Al Ing. Fernando Romero Director del proyecto SIV 25 de la Escuela Superior Politécnica del Chimborazo por haber confiado para realización del componente flora.

\section{Bibliografía}

Azócara, A. (1981). La flora de los páramos. En: Los páramos venezolanos. De Karl Weidman. Edi.Librería Alemena S.R.L. pp. 6-27. Disponible en: http://www.ciens.ula.ve/icae /publicaciones/paramo/articulos_por_autorb.php

Beltrán, K., S. Salgado, F. Cuesta., S. León-Yánez, K. Romoleroux, E. Ortiz, A. Cárdenas y A. Velástegui. (2009). Distribución Espacial, Sistemas Ecológicos y Caracterización Florística de los Páramos en el Ecuador. EcoCiencia, Proyecto Páramo Andino y Herbario QCA. Quito.

Beltrán, K. (2010). Áreas prioritarias para conservación de páramos en la provincia de Chimborazo. En: Bustamante, M., M. Albán y M. Arguello (eds.) (2011). Los páramos del Chimborazo. Un estudio socio-ambiental para la toma de decisiones. Gobierno Autónomo descentralizado de Chimborazo/ EcoCiencia/ CONDESAN/ Programa BioAndes/ Proyecto Páramo Andino. Quito - Ecuador.

Bray, J. R \& , J. T.Curtis. (1957). An ordination of the upland forest communities of southern. Wisconsin. Ecological monographs, 27(4): 325-349

Bustamante, M., M. Albán y M. Arguello (eds.) (2011). Los páramos del Chimborazo. Un estudio socio-ambiental para la toma de decisiones. Gobierno Autónomo descentralizado de Chimborazo/ EcoCiencia/ CONDESAN/ Programa BioAndes/ Proyecto Páramo Andino. Quito - Ecuador.

Caranqui, J., W.Haro y F.Salas. (2013). Diversidad y Similitud de los Páramos del Chimborazo. GADP Chimborazo- ESPOCH. Informe final. 10 pag.

Deguignet, M., Juffe-Bignoli, D., Harrison, J., MacSharry, B., Burgess, N., \& Kingston, N. (2014). United Nations List of Protected Areas. (U. UNEP-WCMC: Cambridge, Ed.) En: Lozano,

P., \& Castro, K. (2015). Evaluación del potencial interpretativo para el aprovechamiento turístico de los sitios destinados a la pesca vivencial de las áreas protegidas de Galápagos. 
European Scientific Journal, XI(20), 63-65. Recuperado el 28 de Agosto de 2015, de http://eujournal.org/index.php/esj/article/view/5953/5739.

EcoCiencia. (2014). Actualización del Plan de Manejo de la Reserva de Producción de Fauna

Chimborazo. Informe final de consultoría.

Eguiguren, P., T. Ojeda, \& N. A. Aguirre. (2010). Diversidad florística del ecosistema páramo del Parque Nacional Podocarpus para el monitoreo del cambio climático. Disponible en: http://www.unl.edu.ec/miccambio/wp-content/uploads/2010/07/Eguiguren-Ojeda 2010 Diversidad-Flor\%C3\%ADstica-del-PNP docx.pdf

Golicher, D . (2012). ¿Cómo cuantificar la diversidad de especies?. Tomado de: http://www.dfpd.edu.uy/cerp/cerp_norte/cn/Biologia/BIODIV/Como\%20cuantificar\%20la\%20d iversidad,\%20algunos\%20ejercicios.pdf

Hofstede, R., Calles, J., López, V., Polanco, R., Torres, F., Ulloa, J., . . . Cerra, M. (2014). Los Páramos Andinos ¿Qué sabemos? Estado de conocimiento sobre el impacto del cambio climático en el ecosistema páramo. UICN. Quito - Ecuador.

Josse, C., P. Mena, y G. Medina. (2000). La Biodiversidad de los Páramos. Serie Páramo 7. GTP. Disponible en: http://paramosecuador.org.ec

Jørgensen, P.M. y S. León-Yánez (Eds.). (1999). Catalogue of the Vascular Plants of Ecuador. Missouri Botanical Garden.

Laaegard S. (1992). Influence of fire in the grass páramo vegetation of Ecuador. 151-170. In: H.Balslev \& J.Luteyn (eds.). Páramo- An Andean Ecosystem under Human Influence. Academic Press London.

León-Yánez, S. (2000). La flora de los páramos ecuatorianos. En: La biodiversidad de los páramos. Serie Páramo 7: 5-21. GTP/AbyaYala. Quito.

León-Yánez, S. (2011). Páramo. Paisaje estudiado, habitado, manejado e institucionalizado. La flora de los páramos ecuatorianos. En: Hofstede, R., Calles, J., López, V., Polanco, R., Torres, F., Ulloa, J., . . Cerra, M. (2014). Los Páramos Andinos ¿Qué sabemos? Estado de conocimiento sobre el impacto del cambio climático en el ecosistema páramo. UICN. Quito Ecuador.

Mena, P. \& R. Hofstede. ( 2006). Los páramos ecuatorianos. Botánica Económica de los Andes Centrales., 91-109.

Ministerio de Ambiente del Ecuador. (1992). Plan General Reserva Chimborazo. Quito- Ecuador. 
Ministerio de Ambiente del Ecuador. (2013). Sistema de Clasificación de los Ecosistemas del Ecuador Continental. Subsecretaría de Patrimonio Natural. Quito.

Missouri Botanical Garden. Trópicos base de datos. [Consulta de internet 31 jul. 2015] http://www.tropicos.org

Pauli H., M. Gottfried, D. Hohenwallner, K. Reiter, R. Casale, G. Grabherr. 2003. Manual para el trabajo de campo del proyecto GLORIA. Instituto de ecología y conservación biológica. Universidad de Viena. Disponible en: http://www.gloria.ac.at/downloads/GLORIA_MS4_Web_espanol.pdf (Consultado julio 10 del 2013)

Rodríguez, M. (2011). Estudio de la diversidad florística a diferentes altitudes en el páramo de almohadillas de la comunidad de Yatzaputzan, cantón Ambato. ESPOCH. Tesis de grado. Escuela de Ingeniería Forestal. Riobamba - Ecuador.

Rojas, S. (2010). Informe técnico del mapa de cobertura vegetal y uso del suelo. Pp: 23. En: Bustamante, M., M. Albán y M. Arguello (eds.) (2011). Los páramos del Chimborazo. Un estudio socio-ambiental para la toma de decisiones. Gobierno Autónomo descentralizado de Chimborazo/ EcoCiencia/ CONDESAN/ Programa BioAndes/ Proyecto Páramo Andino. Quito - Ecuador.

Salgado, S., R. Célleri, S. Rojas, M. Albán, D. Cárate y K. Beltrán. (2011). Caracterización de los páramos de la Provincia de Chimborazo. Pp.: 39-53. En: Bustamante, M., M. Albán y M. Arguello (eds.). Los páramos del Chimborazo. Un estudio socio-ambiental para la toma de desiciones. Gobierno Autónomo descentralizado de Chimborazo/ EcoCiencia/ CONDESAN/ Programa BioAndes/ Proyecto Páramo Andino. Quito.

Salgado, S. y D. Cárate. (2010). Estado de conservación del páramo de pajonal de la provincia de Chimborazo. Pp: 23. En: Bustamante, M., M. Albán y M. Arguello (eds.) (2011). Los páramos del Chimborazo. Un estudio socio-ambiental para la toma de decisiones. Gobierno Autónomo descentralizado de Chimborazo/ EcoCiencia/ CONDESAN/ Programa BioAndes/ Proyecto Páramo Andino. Quito - Ecuador.

Sierra, R. (1999). Propuesta preliminar de un sistema de clasificación de vegetación para el Ecuador continental. Quito - Ecuador.

Sklenář, P., Luteyn, J. L., Ulloa, C., Jørgensen, P. M. y Dillon, M. O. (2005). Flora Genérica de los Páramos. Guía llustrada de las Plantas Vasculares. Memoirs of the New York Botanical Garden, 92, 3-499.

Smith L. \& T. Smith. (2001). Ecología. Pearson Education. Madrid. Pag. 664. Suarez, D., S. 
Chimbolema y T. Paredes. (2012). "Monitoreo del impacto del cambio climático en la biodiversidad de alta montaña en la Región Andina" GLORIA. Corporación Grupo Randi Randi.

Unión Internacional para la Conservación de la Naturaleza. (2008). Directrices para la aplicación de las categorías de gestión de áreas protegidas. (N. Dudley, Ed.) En: Lozano, P., \&

Castro, K. (2015). Evaluación del potencial interpretativo para el aprovechamiento turístico de los sitios destinados a la pesca vivencial de las áreas protegidas de Galápagos. European Scientific Journal, XI(20), 63-65. Recuperado el 28 de Agosto de 2015, de http://eujournal.org/index.php/esj/article/view/5953/5739. 